\title{
Validity of diffusion-weighted magnetic resonance imaging in the evaluation of acute pyelonephritis in comparison with contrast-enhanced computed tomography
}

\author{
Sriman Rajasekaran ${ }^{1 A, B, E, F}$, Venkatesh $K^{1 B}$, Mathew Cheriann ${ }^{1 B}$, Pankaj Mehta ${ }^{1 B}$, Shankar Radhakrishnan ${ }^{2 C, D}$ \\ ${ }^{1}$ Kovai Medical Center and Hospital (KMCH), Coimbatore, India \\ ${ }^{2}$ Vinayaka Mission's Kirupananda Variyar Medical College (VMKVMCH), Salem, India
}

\section{Abstract}

\begin{abstract}
Purpose: Applications of diffusion-weighted magnetic resonance imaging outside the brain have gained increasing importance in recent years, and recent studies have shown the usage of diffusion-weighted (DW) imaging in diagnosing pyelonephritis based on renal cortical and medullary apparent diffusion coefficient (ADC) values. The aim of this study was to assess the validity of DW magnetic resonance (MR) imaging in comparison with contrast-enhanced computed tomography (CECT) in diagnosing pyelonephritis.
\end{abstract}

Material and methods: A cross-sectional observational study was conducted for a period of six months in a tertiary hospital in Coimbatore. All patients with clinical and laboratory diagnosis of acute pyelonephritis, who were referred for radiological imaging (CECT), were taken into the study. Out of 112 patients with a clinical and laboratorial diagnosis of acute pyelonephritis (APN), who underwent both DW MR and CECT, diagnosis of APN was made in 100 patients based on CECT, while in 12 cases the investigation (CECT) was negative. Finally, these 100 patients were included in the study. The validity of DW MR imaging in diagnosing APN was assessed by deriving sensitivity, specificity, and positive and negative predictive value in comparison with CECT findings.

Results: The validity report of DW MR imaging in the detection of APN showed a very high sensitivity (96-100\%) and specificity (86-90\%) and very low false positives (6-10\%) and negatives $(<5 \%)$, and it also showed that in the areas of affected renal parenchyma ADC values were consistently lower compared to unaffected renal parenchyma. Conclusion: Based on the generated hypothesis, DW MR imaging of the kidneys seems to be highly sensitive and specific for the detection of focal or diffuse infections within the kidney in comparison with CECT.

Key words: acute pyelonephritis, CECT imaging, DW MR imaging, ADC value.

\section{Introduction}

Urinary tract infection in the form of infection of the kidneys is a very common disease which involves either the renal parenchyma alone (nephritis) or along with the renal pelvis (pyelonephritis) [1]. Both these conditions when left untreated can lead to renal scarring, which can finally end up with chronic renal failure and hypertension. The diagnosis of urinary tract infection is usually made based on clinical presentations like fever and pain over the loin region and confirmation by conducting a urine analysis with culture. After various considerations, a general consensus was reached for the definition of "complicated" and "non-complicated" acute pyelonephritis (APN) [2,3]. The radiologists' importance is felt in evaluating high-risk patients and to assess the extent of renal involvement, including the presence of abscesses [4]. Contrast-enhanced computed tomography (CECT) studies remains the gold standard technique of radiological examination, which typically show perinephric stranding, enlarged kidneys, and irregular contrast agent

Correspondence address:

Dr. R. Shankar, Professor, No. 413, 20 Feet Road, Peramanur, Salem - 636007, India, e-mail: shnkr_radhakrishnan@yahoo.com

Authors' contribution:

A Study design · B Data collection · C Statistical analysis · D Data interpretation · E Manuscript preparation · F Literature search · G Funds collection 
uptake by the affected kidneys [5]. Although contrast-enhanced magnetic resonance (CEMR) examination is much more accurate, considering its time consumption and the diagnostic accuracy of CECT, which is almost in par with CEMR imaging, MR studies are rarely performed in the acute setting of infectious renal disease [6].

Applications of diffusion-weighted magnetic resonance (DW MR) imaging outside the brain have gained increasing importance in recent years. Extracranial application of diffusion-weighted imaging has been made feasible by a series of technological advances in magnetic resonance imaging, which include faster imaging techniques with echo-planar imaging and parallel imaging, high performance gradients, phased-array multichannel surface coils, and clinical use of higher magnetic field strengths [7]. The role of DW imaging in renal imaging was mostly used in differentiating benign and malignant lesions, but recently the application of DW imaging has also been seen outside the oncological arena. Recent studies have shown the usage of DW imaging in diagnosing pyelonephritis based on the renal cortical and medullary apparent diffusion coefficient (ADC) values, which were found to be lower than those of the unaffected portions of kidneys [8-12]. It has been shown that parenchymal $\mathrm{ADC}$ values in chronic renal failure are significantly lower in both the cortex and medulla than the ADC values of healthy renal parenchyma [13]. However, most of the studies so far conducted on diffusion-weighted MR imaging on the renal system used $1.5 \mathrm{~T}$ and very few studies were conducted with $3.0 \mathrm{~T}$, and so the present study was undertaken to assess the efficacy of DW MR imaging in diagnosing pyelonephritis with $3.0 \mathrm{~T}$.

The aim of this study was to assess the validity of DW MR imaging in comparison with CECT in diagnosing acute uncomplicated pyelonephritis.

\section{Material and methods}

A cross-sectional observational study was conducted for a period of six months in a tertiary hospital in Coimbatore.

Table 1. Age and gender distribution of the study subjects

\begin{tabular}{|c|c|c|c|c|}
\hline \multirow[t]{2}{*}{ Age group } & \multicolumn{2}{|c|}{ Gender, $n(\%)$} & \multirow[t]{2}{*}{ Total, $n(\%)$} & \multirow[t]{2}{*}{$p$ value } \\
\hline & Male & Female & & \\
\hline$<20$ & 0 & $2(3.5)$ & $2(2)$ & \multirow[t]{7}{*}{0.214} \\
\hline $20-30$ & $4(9)$ & $10(17.8)$ & $14(14)$ & \\
\hline $31-40$ & $7(15.9)$ & $4(7.1)$ & $11(11)$ & \\
\hline $41-50$ & $12(27.2)$ & $11(19.6)$ & $23(23)$ & \\
\hline $51-60$ & $8(18.1)$ & $16(28.5)$ & $24(24)$ & \\
\hline $61-70$ & $10(22.7)$ & $10(17.8)$ & $20(20)$ & \\
\hline$>70$ & $3(6.8)$ & $3(5.3)$ & $6(6)$ & \\
\hline Total & $44(100)$ & $56(100)$ & $100(100)$ & \\
\hline Mean \pm SD & $50 \pm 13.5$ & $48.5 \pm 16.1$ & & \\
\hline
\end{tabular}

The study was approved by the institutional Ethics Committee, and informed consent was obtained from each study subject. All patients with clinical and laboratory diagnosis of acute pyelonephritis were considered for the study. Patients who had contraindications for MR and with complicated APN were excluded from the study. A total of 112 patients were taken as the study sample, and all of these 112 patients were subjected to CECT, in which 100 patients the CECT report was suggestive of APN - only those 100 were included in our study. All the 100 patients were subjected to both CECT and DW MR imaging. CECT scanning was performed with GE Light Speed VCT 64-slice scanner at $120 \mathrm{kvp}$ (rotation time $0.8 \mathrm{~s}$, detector coverage $-40.0 \mathrm{~mm}$, helical thickness $0.625 \mathrm{~mm}$ ). Patients were given IV contrast (350 mg) of $1.5 \mathrm{ml} / \mathrm{kg}$ with overall dose ranging from 80 to $100 \mathrm{ml}$ and with an injection rate of $3.5 \mathrm{ml} / \mathrm{s}$ according to the protocol. APN focus was identified as an area of perfusion abnormality shown by: 1) wedge-shaped zones of low attenuation usually extending from papilla to renal capsule and 2) contrast material staining in the previously hypoenhancing zones (characteristic striated nephrogram) at 2-3 h delayed scans.

Then DW MR 3T images were obtained in axial orientation with routinely used $b$ values of $0 / 400 / 800 \mathrm{~s} / \mathrm{mm}^{2}$, and automated ADC (apparent diffusion coefficient) parameter maps were generated by the MR systems. TR/TE - 6400/72, FOV $(\mathrm{mm} \times \mathrm{mm})-380 \times 308$, matrix -192 $\times 125$, slice thickness $-4 \mathrm{~mm}$, number slices -38 , acquisition time - $3 \mathrm{~min} 20 \mathrm{~s}$, parallel imaging - GRAPPA 2 , respiratory control - free breathing, averages -3 , and bandwidth (Hz/px) - 1736. DW imaging assessment was based on sequences of appearance of inflammatory foci as areas of reduced diffusivity with high signal on DW imaging and low ADC values compared to normal renal parenchyma, and the quantitative assessment was done by measuring the ADC by placing circular ROI in the pathological renal parenchyma.

All data were entered and analysed using SPSS version 24. The validity of DW MR imaging in diagnosing APN was assessed by deriving sensitivity, specificity, and positive and negative predictive values in comparison with CECT findings.

\section{Results}

The demographic details of the study subjects show that the majority of them are females with a male:female ratio of $0.72: 1$, and most of them were in the age group between 50 and 70 years with a mean age of 55 and 50 years among males and females, respectively (Table 1). As already described in the methodology, all the patients with acute pyelonephritis were subjected to both CECT and DW MR imaging, most of the infected foci that were picked up by CECT were also identified by DW MR imaging, all the three poles of both right and left kidneys were 
Table 2. Detection of acute pyelonephritis by CECT and DW MR imaging

\begin{tabular}{|l|c|c|c|c|c|c|}
\hline \multirow{2}{*}{ Imaging technique } & \multicolumn{3}{|c|}{ Right kidney $(n=100)$} & \multicolumn{3}{c|}{ Left kidney $(n=100)$} \\
\cline { 2 - 7 } & Upper & Middle & Lower & Upper & Middle & Lower \\
\hline CECT & 54 & 44 & 50 & 56 & 60 & 46 \\
\hline DW MR & 52 & 44 & 48 & 54 & 58 & 46 \\
\hline$p$ value & 0.981 & 1.000 & 0.923 & 0.931 & 0.918 & 1.000 \\
\hline
\end{tabular}

CECT - contrast-enhanced computed tomography, DW MR - diffusion-weighted magnetic resonance, $p$ value derived by applying $\chi^{2}$ test

Table 3. Validity of DW MR imaging in comparison with CECT in detection of APN

\begin{tabular}{|l|c|c|c|c|c|c|}
\hline Site of APN & Sensitivity & Specificity & $\begin{array}{c}\text { Positive } \\
\text { predictive value }\end{array}$ & $\begin{array}{c}\text { Negative } \\
\text { predictive value }\end{array}$ & $\begin{array}{c}\text { Percentage } \\
\text { of false positive }\end{array}$ & $\begin{array}{c}\text { Percentage } \\
\text { of false negative }\end{array}$ \\
\hline Right upper pole & $96.6 \%$ & $88.9 \%$ & $89.6 \%$ & $95.2 \%$ & $10.3 \%$ & $4.7 \%$ \\
\hline Right middle pole & $100 \%$ & $90.2 \%$ & $88 \%$ & $100 \%$ & $12 \%$ & $0 \%$ \\
\hline Right lower pole & $96 \%$ & $92 \%$ & $92.3 \%$ & $95.8 \%$ & $7.6 \%$ & $4.1 \%$ \\
\hline Left upper pole & $96.4 \%$ & $89.6 \%$ & $90 \%$ & $95 \%$ & $10 \%$ & $5 \%$ \\
\hline Left middle pole & $96.6 \%$ & $90 \%$ & $93.5 \%$ & $94.7 \%$ & $6.4 \%$ & $5.2 \%$ \\
\hline Left lower pole & $100 \%$ & $90.2 \%$ & $88.4 \%$ & $100 \%$ & $11.5 \%$ & $0 \%$ \\
\hline
\end{tabular}

APN - acute pyelonephritis, CECT - contrast-enhanced computed tomography, DW MR - diffusion-weighted magnetic resonance.

equally affected, and there was no statistical significant difference in identification of APN between CECT and DW MR imaging (Table 2). The validity of DW MR imaging in detecting APN was assessed by deriving sensitivity, specificity, positive and negative predictive values, and percentages of false positive and false negative. A newer diagnostic technique is considered to be highly valid when its sensitivity, specificity, and positive and negative predictive value is more than $90 \%$ and the percentage of false positive and negative is less than $10 \%$. The validity report of DW MR imaging shows a very high sensitivity (96-100\%) and specificity (86-90\%) and a very low false positives $(6-10 \%)$ and negatives $(<5 \%)$ (Table 3$)$. The detection of APN by CECT images and its corresponding ADC mapping by DW MR imaging is shown in Figure 1, he detection of renal abscess is seen in Figure 2, and the DW imaging sequence of all three poles in a case of acute pyelonephritis is shown in Figure 3. It was clearly shown that the areas of affected renal parenchyma $\mathrm{ADC}$ value in $\mathrm{mm}^{2} / \mathrm{s}$ was found to be consistently lower. The ADC value of the affected foci ranged between 1143.30 and $1309.81\left(10^{-6} \mathrm{~mm}^{2} / \mathrm{s}\right)$ (Table 4$)$. In our study we had nine patients with renal abscess, and these abscesses were easily detected on DW MR imaging, especially with higher $b$ values. The ADC values in kidney abscesses were lower than the APN lesions. ADC values in the abscessed area ranged from 651.51 to $943.45 \times 10^{-6} \mathrm{~mm}^{2} / \mathrm{s}$. The ADC in the non-affected renal parenchyma consistently ranged between $1900 \pm 200 \times 10^{-6} \mathrm{~mm}^{2} / \mathrm{s}$ in all the patients included in our study, which was reaffirmed by other studies,


Figure 1. A) Contrast-enhanced computed tomography shows areas of reduced attenuation (arrow) during the nephrographic phase on left side. B) Corresponding ADC map appear hypointense (arrow) (mean ADC of the infected foci is $1173 \times 10^{-6} \mathrm{~mm}^{2} / \mathrm{s}$ ) 



Figure 2. 43-year-old patient with acute pyelonephritis (APN). Small non-enhancing hypo density (arrow - no significant change in $\mathrm{HU}$ ) is seen in the posterior cortex of left midpole region (arrow) - suggestive of renal abscess ( $\mathbf{A}$ - plain, $\mathbf{B}$ - arterial phase, $\mathbf{C}$ - venous phase). 0 n axial diffusion-weighted imaging of the same patient the lesion appears progressively hyperintense with increasing b values (D, E, F, respectively). Apparent diffusion coefficient map (G) demonstrates hypointense signal with markedly decreased ADC values as compared to the APN lesions (mean ADC in the lesion measures $A D C$ of $669 \times 10^{-6} \mathrm{~mm}^{2} / \mathrm{s}$ ) 

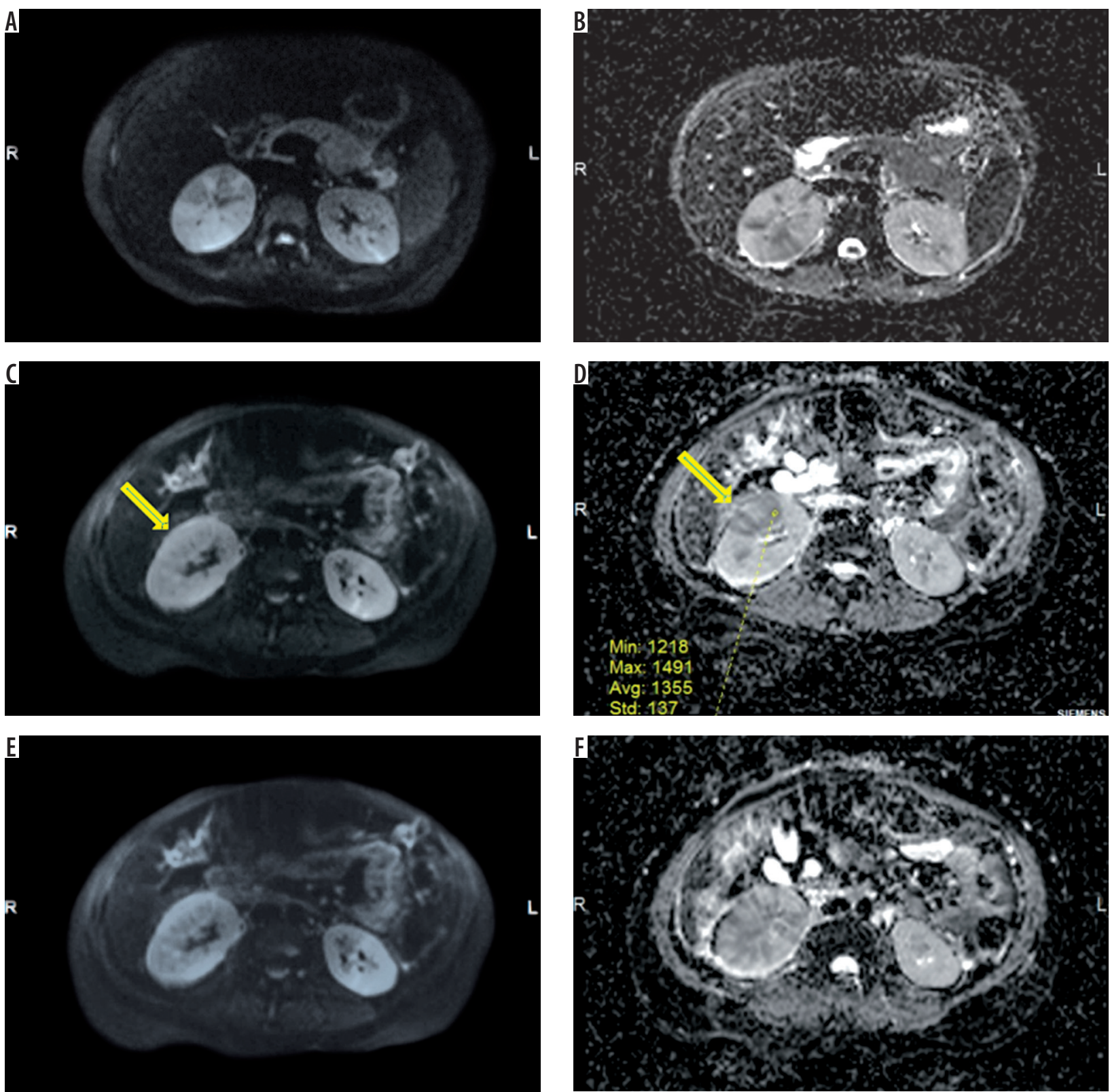

Figure 3. Acute right pyelonephritis. Axial diffusion-weighted (DW) imaging sequences at three levels ( $A, B$ - upperpole, C, D - midpole, E, F-lower pole) in the right kidney reveal heterogeneously hyperintense lesions with corresponding hypointense signal in $A D C$ map. The mean $A D C$ of one of the inflammatory foci (arrows) in midpole is $1355 \times 10^{-6} \mathrm{~mm}^{2} / \mathrm{s}$ (D). The involvement of lower pole in this patient was subtle on contrast-enhanced computed tomography but obvious on DW imaging

and a statistically significant difference $(p<0.05)$ was observed in the ADC values between normal and affected renal parenchyma (Table 4 ).

\section{Discussion}

Acute pyelonephritis is one of the most common urological diseases, which, when left undiagnosed, leads to severe complications like CRF. This is where imaging plays a vital role and is needed to assess the severity of kidney involvement and to plan the further course of therapy.

Both CT and MR imaging provide excellent image quality, which helps in the diagnostic work-up of renal infections. Imaging of the kidneys is challenging because
Table 4. Mean, SD, and 95\% Cl of ADC values of the infected foci represented in $10^{-6} \mathrm{~mm}^{2} / \mathrm{s}$

\begin{tabular}{|l|c|c|c|c|}
\hline Parameter & Mean & SD & $95 \%$ Cl & \multirow{2}{*}{$p$ value } \\
\hline Right upper pole & 1245.52 & 118.84 & $1215.26-1276.18$ & $<0.0001$ \\
\hline Right middle pole & 1255.44 & 125.26 & $1221.18-1289.87$ & \\
\cline { 1 - 4 } Right lower pole & 1231.45 & 91.58 & $1206.45-1255.78$ & \\
\cline { 1 - 4 } Left upper pole & 1217.34 & 89.24 & $1196.89-1239.72$ & \\
\cline { 1 - 4 } Left middle pole & 1202.77 & 97.53 & $1178.54-1224.56$ & \\
\cline { 1 - 4 } Left lower pole & 1225.44 & 104.18 & $1197.08-1254.19$ & \\
\cline { 1 - 4 } Normal value & 1900 & 200 & - & \\
\hline
\end{tabular}


not all patients can undergo contrast-enhanced examinations. Administration of contrast medium is limited to patients who have normal renal function. A well-known complication in patients with impaired renal function who are subjected to CECT is contrast-induced nephropathy. Also, because of the reports on nephrogenic systemic fibrosis in recent years, contrast medium administration for MR imaging must also be avoided in this patient group. This is where the non-invasive DW MR imaging plays a pivotal role in eliminating the risk associated with contrast agents [14].

Many of the latest studies have shown the role of DWMRI in the assessment of various renal diseases (that includes renal ischaemia, pyonephrosis, and diffuse renal disease), its role in the diagnosis and follow-up of the APN is increasing. A noticeable characteristic of DW imaging is that in the source data with high $b$ values (i.e. $b=800 \mathrm{~s} / \mathrm{mm}^{2}$ ) even the smallest foci of infection are displayed with high lesion-to-background contrast [15-20]. This high conspicuity of inflammatory changes in combination with the assumed high sensitivity could foster the use of DW imaging as a primary tool for workup of complicated patients or patients with impaired renal function.

The validity of DW MR imaging in our study showed a very high sensitivity and specificity with a very low percentage of false positive and false negative. The potential reason for the few false positives in the study is that the hyperintense signal detected on DW MR imaging may represent persistent moderate inflammatory events of varying severity, which might have gone undetected by CECT. A study conducted by Vivier et al. compared DW imaging with gadolinium-enhanced T1-weighted imaging among paediatric patients with suspicion of APN, and the results proved that DW imaging provided comparable results to gadolinium-enhanced T1-W MR imaging in diagnosis of acute pyelonephritis, and so the contrast medium injection could be effectively avoided [21]. Another study done by Henninger et al. on the value of DW MR imaging for the detection of nephritis on 21 patients showed that DW imaging of the kidneys seems to be highly sensitive for the detection of infections within the kidney [22].

The diffusion restriction in DW imaging can be absolutely quantified by means of the ADC. This is routinely done in oncological imaging in order to investigate the malignant potential of the tumour. In this study ADC values of infected renal tissue were significantly lower than the ADC values of healthy renal tissue. Hence, a safe differentiation of infection from malignant tumours with atypical infiltrative growth pattern such as transitional cell carcinomas does not seem feasible based on the ADC values only, which is one of the limitations of DWI MR imaging. This can be overcome by incorporating the T2 HASTE sequence to rule out any gross structural pathologies.

Computed tomography of infectious renal disease is still the mainstay of imaging in radiology. There is a wealth of knowledge on CT appearances of various rare renal disease conditions such as papillary necrosis or emphysematous pyelonephritis and xanthogranulomatous pyelonephritis. While these conditions might also be detected on MR imaging, the detectability of air and calcifications is much higher in CT [23]. In particular, the potential to display renal calculi with high accuracy and at low radiation dose triggers a large amount of CT exams of the urogenital system [24]. Apart from availability, which is still limited for MR imaging at many sites, the clinical questions regarding "urolithiasis" and "gas-forming infection" will further require a CT study to be performed.

\section{Conclusions}

Based on the hypothesis-generated DW MR imaging of the kidneys seems to be highly sensitive and specific for the detection of focal or diffuse infections within the kidney in comparison with CECT, with an added advantage of not using ionising radiation or contrast media. This is especially useful in paediatric patients, pregnant women, patients with impaired renal function, and patients with previous allergic history to contrast with clinical suspicion of APN. Through the present study we can recommend an MRI protocol for acute pyelonephritis, which would be of two components: a) T2 HASTE - to rule out any gross structural abnormalities and b) DW MR imaging with ADC to look for infectious foci. This protocol has the possibility to make MR imaging cheaper and less time consuming in this setting, which could revolutionise diagnostic imaging in the evaluation of APN. However largescale prospective studies are warranted to further establish the role of DW imaging in diagnosing APN.

\section{Conflict of interest}

The authors declare no conflict of interest.

\section{References}

1. Foxman B. Epidemiology of urinary tract infections: incidence, morbidity, and economic costs. Am J Med 2002; 113 (suppl 1A): 5S-13S.

2. Dyer RB. CT of renal inflammatory disease. Invited commentary. Radiographics 1997; 17: 867-868.

3. Soulen MC, Fishman EK, Goldman SM, Gatewood OM. Sequelae of acute renal infection: CT evaluation. Radiology 1989; 173: 423-426.
4. Nicolle LE. The chronic indwelling catheter and urinary infection in long-term-care facility residents. Infect Control Hosp Epidemiol 2001; 22: 316-321.

5. Kawashima A, Sandler CM, Goldman SM, Raval BK, Fishman EK. CT of renal inflammatory disease. Radiographics 1997; 17: 851-866. 
6. Niemann T, Kollmann T, Bongartz G. Diagnostic performance of low-dose CT for the detection of urolithiasis: a meta-analysis. Am J Roentgenol 2008; 191: 396-401.

7. Le Bihan D, Breton E, Lallemand D, Grenier P, Cabanis E, LavalJeantet M. MR imaging of intravoxel incoherent motions: application to diffusion and perfusion in neurologic disorders. Radiology 1986; 161: 401-407.

8. Müller MF, Prasad P, Siewert B, Nissenbaum MA, Raptopoulos V, Edelman RR. Abdominal diffusion mapping with use of a wholebody echo-planar system. Radiology 1994; 190: 475-478.

9. Chow LC, Bammer R, Moseley ME, Sommer FG. Single breath-hold diffusion-weighted imaging of the abdomen. J Magn Reson Imaging 2003; 18: 377-382.

10. Yamashita Y, Tang Y, Takahashi M. Ultrafast MR imaging of the abdomen: echo planar imaging and diffusion-weighted imaging. J Magn Reson Imaging 1998; 8: 367-374.

11. Yoshikawa T, Kawamitsu H, Mitchell DG, et al. ADC measurement of abdominal organs and lesions using parallel imaging technique. AJR Am J Roentgenol 2006; 187: 1521-1530.

12. Matsuoka A, Minato M, Harada M, et al. Comparison of 3.0- and 1.5-tesla diffusion-weighted imaging in the visibility of breast cancer. Radiat Med 2008; 26: 15-20.

13. Verswijvel G, Vandecaveye V, Gelin G, et al. Diffusion-weighted MR imaging in the evaluation of renal infection: preliminary results. JBR-BTR 2002; 85: 100-103.

14. Thoeny HC, Binser T, Roth B, Kessler TM, Vermathen P. Noninvasive assessment of acute ureteral obstruction with diffusion-weighted MR imaging: a prospective study. Radiology 2009; 252: 721-728.

15. Yang D, Ye Q, Williams DS, Hitchens TK, Ho C. Normal and transplanted rat kidneys: diffusion MR imaging at 7 T. Radiology 2004; 231: 702-709.
16. Thoeny HC, Zumstein D, Simon-Zoula S, et al. Functional evaluation of transplanted kidneys with diffusion-weighted and BOLD MR imaging: initial experience. Radiology 2006; 241: 812-821.

17. Yamanouchi Y, Yamamoto K, Noda K, Tomori K, Kinoshita T. Renal infarction in a patient with spontaneous dissection of segmental arteries: diffusionweighted magnetic resonance imaging. Am J Kidney Dis 2008; 52: 788-791.

18. Reinig JW, Doppman JL, Dwyer AJ, Frank J. MRI of indeterminate adrenal masses. AJR Am J Roentgenol 1986; 147: 493-496.

19. Hönigschnabl S, Gallo S, Niederle B, et al. How accurate is MR imaging in characterisation of adrenal masses: update of a long-term study. Eur J Radiol 2002; 41: 113-122.

20. Tsushima Y, Takahashi-Taketomi A, Endo K. Diagnostic utility of diffusion-weighted MR imaging and apparent diffusion coeffi cient value for the diagnosis of adrenal tumors. J Magn Reson Imaging 2009; 29: 112-117.

21. Vivier PH, Sallem A, Beurdeley M, et al. MRI and suspected acute pyelonephritis in children: comparison of diffusion-weighted imaging with gadolinium-enhanced T1-weighted imaging. Eur Radiol 2014; 24: 19-25.

22. Henninger B, Reichert M, Haneder S, Schoenberg SO, Michaely HJ. Value of diffusion-weighted MR imaging for the detection of nephritis. ScientificWorldJournal 2013; 2013: 348105.

23. Grayson DE, Abbott RM, Levy AD, Sherman PM. Emphysematous infections of the abdomen and pelvis: a pictorial review. Radiographics 2002; 22: 543-561.

24. Worster A, Preyra I, Weaver B, Haines T. The accuracy of noncontrast helical computed tomography versus intravenous pyelography in the diagnosis of suspected acute urolithiasis: a meta-analysis. Ann Emerg Med 2002; 40: 280-286. 